\title{
Lower Bound on Minimum Lee Distance of Algebraic-Geometric Codes over Finite Fields
}

\author{
Xin-Wen $\mathbf{W u}^{\star}$, Margreta Kuijper ${ }^{\dagger}$ and Parampalli Udaya ${ }^{\ddagger}$ \\ * School of Information Technology and Mathematical Sciences \\ University of Ballarat, VIC 3353, Australia \\ Email: x.wu@ballarat.edu.au \\ $\dagger$ Department of Electrical and Electronic Engineering \\ University of Melbourne, VIC 3010, Australia \\ Email: m.kuijper@ee.unimelb.edu.au \\ $\ddagger$ Department of Computer Science and Software Engineering \\ University of Melbourne, VIC 3010, Australia \\ Email: udaya@csse.unimelb.edu.au
}

\begin{abstract}
We study algebraic-geometric (AG) codes over finite fields with respect to the Lee metric. A lower bound on the minimum Lee distance is derived, which is a Lee-metric version of the well-known Goppa bound on the minimum Hamming distance of AG codes. The bound generalizes a lower bound on the minimum Lee distance of Lee-metric BCH and Reed-Solomon codes, which have been successfully used for protecting against bitshift and synchronization errors in constrained channels and for the error control in partial-response channels.
\end{abstract}

\section{Introduction}

For block codes over $G F(p)$, where $p$ is an odd prime, it has been found $[1,2]$ that for many applications where non-binary signals are transmitted or stored the Lee metric is a more appropriate metric than the Hamming metric. For $\alpha \in G F(p)$, denote by $\bar{\alpha}$ the smallest nonnegative integer such that $\alpha=\bar{\alpha} \cdot 1$, where 1 is the multiplicative unity of $G F(p)$. The Lee value $|\alpha|$ of $\alpha$ is defined as

$$
|\alpha|= \begin{cases}\bar{\alpha}, & \text { when } 0 \leq \bar{\alpha} \leq(p-1) / 2 \\ p-\bar{\alpha}, & \text { when }(p+1) / 2 \leq \bar{\alpha} \leq p-1\end{cases}
$$

For $\mathbf{c}=\left(c_{1}, c_{2}, \cdots, c_{n}\right) \in G F(p)^{n}$, the Lee weight is defined as $\|\mathbf{c}\|=\sum_{i=1}^{n}\left|c_{i}\right|$. The Lee distance between two vectors is defined as the Lee weight of their difference. The minimum Lee distance of a block code over $G F(p)$ is the minimum value of all the Lee distances between any pair of distinct codewords. The minimum Lee distance is one of the most important code parameters that determines the Lee-errorcorrecting capability. 
$\mathrm{BCH}$ codes over $G F(p)$ with respect to the Lee metric and their applications to constrained and partial-response channels are studied in [2]. Lower bounds on the minimum Lee distance of BCH codes are derived and a Lee-metric decoding algorithm is presented. Reed-Solomon (RS) codes are a class of block codes which are frequently used in various error-control systems. The lower bounds in [2] also serve as lower bounds on the minimum Lee distance of $\mathrm{RS}$ codes over $G F(p)$, as $\mathrm{RS}$ codes can be viewed as subcodes of the $\mathrm{BCH}$ codes in [2].

Algebraic-geometric codes (AG codes for short) over finite fields [3, 4] form a large family of linear codes, which includes RS codes, Reed-Muller codes and Goppa codes as special instances. The code parameters of AG codes (i.e., length, dimension, and minimum distance) with respect to the Hamming metric have been extensively studied in the literature. In particular, the Goppa bound is a well-known lower bound on the minimum Hamming distance of AG codes [3, 4].

In this paper we are interested in properties of $\mathrm{AG}$ codes with respect to the Lee metric. In particular, we seek to generalize the results on $\mathrm{BCH}$ and RS codes in [2]. Apart from being theoretically interesting this will also help the application of AG codes to constrained and partial-response channels.

More specifically, in this paper we derive a lower bound on the minimum Lee distance of AG codes. Our bound is a generalization of a lower bound on the minimum Lee distance of BCH codes (Theorem 4 in [2]). As we will explain in the following sections, the proof of our bound is based on a property of rational functions (Property $\mathrm{A}$ in the next section) that was used in [3, 4] to prove the Goppa bound for AG codes with respect to the Hamming metric. Thus, our bound can also be viewed as a Lee-metric version of the Goppa bound. The bound has been reported at conferences [5,6] along with some of our results on Lee-metric decoding of AG codes.

\section{Algebraic-Geometric Codes}

Let $\mathcal{X}$ be a nonsingular, absolutely irreducible curve in $\mathbf{P}_{F}^{m}$, the $m$-dimensional projective space over a field $F$. Denote by $F(\mathcal{X})$ the function field of $\mathcal{X}$ over $F$. Let $P$ be a closed point of $\mathcal{X}$. We denote by $\nu_{P}(\cdot)$ the discrete valuation associated with $P$. If $\nu_{P}(\varphi)>0$, then $\varphi(P)=0$, and we say that $\varphi$ has a zero of order (or multiplicity) $\nu_{P}(\varphi)$ at $P$. If $\nu_{P}(\varphi)<0$, then $\varphi^{-1}(P)=0$, and $\varphi$ is said to have a pole of order (or multiplicity) $-\nu_{P}(\varphi)$ at $P$. For any nonzero rational function $\varphi \in F(\mathcal{X})$ there are only finitely many closed points $P$ such that $\nu_{P}(\varphi) \neq 0$. We can therefore define the principal divisor of $\varphi$ as

$$
\operatorname{div}(\varphi)=\sum \nu_{P}(\varphi) P
$$

where the sum is taken over all closed points. Further, we define the degree of the principal divisor $\operatorname{div}(\varphi)$ as $\operatorname{deg}(\operatorname{div}(\varphi))=\sum \nu_{P}(\varphi)$. The following is a well-known property of rational functions (cf. $[3,4])$. 
Property A For a nonzero rational function $\varphi$, we have that $\operatorname{deg}(\operatorname{div}(\varphi))=0$.

The proof of our lower bound on minimum Lee distance of AG codes will be based on this property.

For a divisor $D$ of $\mathcal{X}$, define $L(D)=\{f \mid f \in F(\mathcal{X}), f=0$ or $\operatorname{div}(f)+D \geq 0\}$. It can be proved that $L(D)$ is a linear space over $F$. For any divisors $D$ and $D^{\prime}$ with $D \geq D^{\prime}$, we have $L(D) \supseteq L\left(D^{\prime}\right)$. Let $\rho$ be a nonnegative integer and $P$ be a point of $\mathcal{X}$. If $L(\rho P) \neq L((\rho-1) P)$, or equivalently if there exists a rational function $\varphi$, such that $\varphi$ has a pole only at $P$ with $\nu_{P}(\varphi)=-\rho$, then we call $\rho$ a nongap of $P$. Let $\left\{\rho_{1}, \rho_{2}, \rho_{3}, \cdots\right\}$ be the set of all nongaps of $P$, ordered as $\rho_{1}<\rho_{2}<\rho_{3}<\cdots$, and let $g$ be the genus of the curve. Then,

$$
0=\rho_{1}<\rho_{2}<\cdots<\rho_{g}<\rho_{g+1}=2 g,
$$

and $\rho_{i}=i+g-1$ when $i \geq g+1$ (see [3]). Let $\varphi_{1}, \varphi_{2}, \varphi_{3}, \cdots$ be a sequence of rational functions, such that $\varphi_{i}$ has a pole only at $P$ and $\nu_{P}\left(\varphi_{i}\right)=-\rho_{i}$. Then $\left\{\varphi_{1}, \varphi_{2}, \ldots, \varphi_{i}\right\}$ is a basis of $L\left(\rho_{i} P\right)$.

Suppose $\left\{P_{1}, P_{2}, \cdots, P_{n}\right\}$ is a set of rational points of a nonsingular, absolutely irreducible curve $\mathcal{X}$ of genus $g$ over $F=G F(q)$. Let $D$ be the divisor $D=P_{1}+\cdots+P_{n}$, and let $G$ be another divisor of $\mathcal{X}$ satisfying $\sup (D) \cap \sup (G)=\emptyset$. An algebraic-geometric $(A G)$ code $C_{L}(D, G)$ over $G F(q)$ is defined as

$$
C_{L}(D, G)=\left\{\left(f\left(P_{1}\right), f\left(P_{2}\right), \cdots, f\left(P_{n}\right)\right) \mid f \in L(G)\right\}
$$

Denote $\rho=\operatorname{deg} G$. If $\rho<n$, then $C_{L}(D, G)$ has length $=n$ and dimension $\geq \rho-g+1$. If moreover $\rho>2 g-2$, then the dimension of this code is $\rho-g+1$.

\section{Lower Bound on the Minimum Lee Distance}

We consider $\mathrm{AG}$ codes $C_{L}(D, \rho P)$ of length $n$ and dimension $\rho-g+1$. For notational convenience, our bound is presented for AG codes over $G F(p)$, where $p$ is an odd prime. However, later in this section, we will explain that the bound can be straightforwardly extended to AG codes over any finite field $G F(q)$ where $q$ is a prime power.

Theorem For an $[n, k=\rho-g+1]$ algebraic-geometric code $C_{L}(D, \rho P)$ over $G F(p)$, the minimum Lee distance $d_{\mathcal{L}}\left(C_{L}(D, \rho P)\right)$ satisfies

$$
d_{\mathcal{L}}\left(C_{L}(D, \rho P)\right) \geq \frac{n^{2}-\rho^{2}}{4 \rho} .
$$

Extending the idea for the Proof of Theorem 4 in [2], we construct a vector which satisfies certain conditions; and then prove that all the nonzero codewords in $C_{L}(D, \rho P)$ have equal or greater Lee weights than this vector. We will use Property A in the previous section in our proof. 
Proof of the Theorem: Any nonzero codeword $\mathbf{c}$ of $C_{L}(D, \rho P)$ has the form $\mathbf{c}=\left(\varphi\left(P_{1}\right), \cdots, \varphi\left(P_{n}\right)\right)$ where $\varphi$ is a nonzero rational function in $L(\rho P)$. By the definition of $L(\rho P)$, the rational function $\varphi$ has no pole except $P$, and the multiplicity of the pole of $\varphi$ at $P$ is at most $\rho$. From Property A, the number of zeros of $\varphi$ is at most $\rho$. Now, let $\alpha$ be any element of $G F(p)$. As $L(\rho P)$ is a linear space over $G F(p)$, we have $\varphi-\alpha \in L(\rho P)$. Therefore, $\varphi-\alpha$ has at most $\rho$ zeros. This implies the following facts:

- The value $\alpha$ can appear in the coordinates of the codeword $\mathbf{c}$ at most $\rho$ times.

- The code length $n \leq \rho p$.

Let $M=\left\lfloor\frac{n-\rho}{2 \rho}\right\rfloor$ and $N=n-\rho-2 \rho M$. By definition, $N$ is the remainder of dividing $n-\rho$ by $2 \rho$. Thus, $N \geq 0$ and $n=\rho+2 \rho M+N$. When $N>0$, we have $M+1<\frac{n-\rho}{2 \rho}+1=\frac{n+\rho}{2 \rho} \leq \frac{p+1}{2}$; thus, as an integer $M+1 \leq \frac{p-1}{2}$. When $N=0$, we have $M=\frac{n-\rho}{2 \rho} \leq \frac{p-1}{2}$.

We construct a word $\mathbf{a}=\left(a_{1}, \cdots, a_{n}\right)$ in $G F(p)^{n}$ satisfying the following conditions:

1) 0 appears in the coordinates of a exactly $\rho$ times;

2) In the coordinates of a, each of the $2 M$ elements of $G F(p)$, namely, $\pm 1, \pm 2, \cdots, \pm M$, appears exactly $\rho$ times;

3) If $N>0$, the remaining $N$ coordinates of a are filled with the elements $M+1$ or $-(M+1)$ of $G F(p)$.

From the construction of $\mathbf{a}$, it is easy to see that for any codeword $\mathbf{c}$, the Lee weight $\|\mathbf{c}\|_{\mathcal{L}} \geq\|\mathbf{a}\|_{\mathcal{L}}$. Now compute the Lee weight of $\mathbf{a}$ as

$$
\|\mathbf{a}\|_{\mathcal{L}}=2 \rho \sum_{i=1}^{M} i+N(M+1)=(\rho M+N)(M+1) .
$$

Denoting $\mu=\frac{n-\rho}{2 \rho}$ and $\gamma=\frac{N}{2 \rho}$, from the definition of $M$ and $N$, we have $0 \leq \gamma<1$ and $\mu-\gamma=M$. Thus,

$$
\begin{aligned}
\|\mathbf{a}\|_{\mathcal{L}} & =(\rho M+N)(M+1) \\
& =(\rho(\mu-\gamma)+2 \rho \gamma)(\mu-\gamma+1) \\
& =\rho\left(\mu^{2}+\mu-\gamma^{2}+\gamma\right) \\
& \geq \rho \mu(\mu+1) \\
& =\left(n^{2}-\rho^{2}\right) /(4 \rho) .
\end{aligned}
$$

Therefore, $d_{\mathcal{L}}\left(C_{L}(D, \rho P)\right) \geq\|\mathbf{c}\|_{\mathcal{L}} \geq \frac{n^{2}-\rho^{2}}{4 \rho}$, which proves the theorem.

Let us recall the well-known Goppa bound on the minimum Hamming distance of AG codes,

$$
d_{H} \geq n-\rho
$$


which is a straightforward corollary of Property A (cf. [3, 4]). As the minimum Lee distance $d_{\mathcal{L}}$ is always greater than or equal to the minimum Hamming distance $d_{H}$, we can also view $n-\rho$ as a lower bound on the minimum Lee distance. However, this lower bound is much looser than the bound in the above theorem. Indeed, it is easy to verify that when $n>3 \rho$, the value of $\left(n^{2}-\rho^{2}\right) /(4 \rho)$ exceeds the value of $n-\rho$.

We observe that the Lee distance can be defined for block codes over any finite field $G F(q)$ where $q$ is a prime power. Denote by $\mathbb{Z}_{q}$ the ring of integers modulo $q$. Let us fix a bijection

$$
\begin{aligned}
G F(q) & \longrightarrow \mathbb{Z}_{q} \\
\beta & \longmapsto \bar{\beta}
\end{aligned}
$$

For any $b \in \mathbb{Z}_{q}$, the Lee value $|b|$ of $b$ is the nonnegative integer $\min \{b, q-b\}$. The Lee value $|\beta|$ of $\beta \in G F(q)$ is then defined as $|\bar{\beta}|$. As the proof of the above theorem is independent of specific structures of finite fields, the lower bound given in the theorem is straightforwardly extended to AG codes over any finite field $G F(q)$.

\section{Conclusion}

We present a lower bound on the minimum Lee distance of AG codes. This bound generalizes a lower bound on the minimum Lee distance of Lee-metric BCH codes. These Lee-metric BCH codes have been successfully used for protecting against bitshift errors and synchronization errors in constrained channels and for the error control in partial-response channels in [2]. The bound can be viewed as a Lee-metric version of the well-known Goppa bound. Apart from being theoretically interesting this result will also be useful in the application of AG codes to constrained and partial-response channels.

Acknowledgement: This work was supported by the Australian Research Council. 


\section{References}

[1] E.R. Berlekamp, Algebraic Coding Theory, Aegean Park Press, 1984.

[2] R.M. Roth and P.H. Siegel, "Lee-Metric BCH Codes and their Application to Constrained and Partial-Response Channels," IEEE Trans. Inform. Theory, vol. 40, no. 4, Jul. 1994, pp. 1083-1096.

[3] I.B. Blake, C. Heegard, T. Høholdt, and V. Wei, "Algebraic-Geometry Codes," IEEE Trans. Inform. Theory, vol. 44, no. 6, Oct. 1998, pp. 2596-2618.

[4] T. Høholdt, J.H. van Lint, and R. Pellikaan, "Algebraic Geometry Codes," In Handbook of Coding Theory, vol.1, (V.S. Pless, W.C. Huffman, and R.A. Brualdi, Eds), Elsevier, Amsterdam, 1998, pp.871-961.

[5] X.-W. Wu, M. Kuijper, and P. Udaya, "Improved Decoding of Algebraic-Geometric Codes with Respect to the Lee Metric," Proceedings of 2005 Australian Communications Theory Workshop, Brisbane, Australia, 2-4 February, 2005.

[6] X.-W. Wu, M. Kuijper, and P. Udaya, "On the Decoding Radius of Lee-Metric Decoding of Algebraic-Geometric Codes," Proceedings of 2005 IEEE International Symposium on Information Theory, Adelaide, Australia, 5 - 9 September, 2005. 